\title{
Pseudomonas aeruginosa Infections: Factors Relating to Mortality with Emphasis on Resistance Pattern and Antimicrobial Treatment
}

\author{
Millena R.S. Pinheiro ${ }^{1,2}$, Heloisa R. Lacerda ${ }^{1,3}$, Renata G.L. Melo ${ }^{2}$ and $\mathbf{M}^{\text {a }}$ Amélia Maciel $^{3}$ \\ ${ }^{1}$ Postgraduate Course in Health Sciences, Federal University of Pernambuco (UFPE); ${ }^{2}$ Infection Control Service, Hospital Prontolinda, Olinda; \\ ${ }^{3}$ Infectious and Parasitic Diseases Clinic, Hospital das Clínicas, Federal University of Pernambuco (UFPE); Recife, PE, Brazil
}

\begin{abstract}
A retrospective case-control study was conducted to investigate the risk factors for death among intensive care unit patients with Pseudomonas aeruginosa infection. Out of 131 patients investigated, 67 (51.1\%) died within 30 days of being diagnosed with this infection. The mean duration of hospital stay before this diagnosis was $28.5 \pm 26.5$ days. No association was found between bacterial resistance and death in this study (multiresistant $p=0.26$; panresistant $\mathrm{p}=\mathbf{0 . 4 2}$ ), but the adequacy of the initial treatment was inversely proportional to the degree of resistance. There was a tendency towards greater mortality among patients who received combination therapy (empirical $p=0.09$; definitive $\mathbf{p}=\mathbf{0 . 0 8}$ ), despite the greater frequency of appropriate treatment in these patients and the similar degree of severity in the two groups. This finding may be explained by pharmacodynamic parameters that were not studied or by the extensive use of aminoglycosides in the combination therapy, which play a controversial role in combination therapy due to their potential for renal toxicity. The multivariate analysis in our study demonstrated that age [odds ratio (OR) 1.04], septic shock (OR 15.4) and hypoalbuminemia (OR 0.32) were independent risk factors for death. Key-Words: Risk factors, case-control study, Pseudomonas aeruginosa, death.
\end{abstract}

Pseudomonas aeruginosa has evolving virulence characteristics and antimicrobial resistance patterns which make it a difficult target for antibiotic therapy [1-3]. It was the most frequently found bacterium in lower airway infections in Brazilian hospitals of the SENTRY study [4], displaying a resistance of $49 \%$ to imipenem and of $49.8 \%$ to ceftazidime. The MYSTIC program carried out in 20 Brazilian hospitals also found that $P$. aeruginosa was the most frequent Gramnegative isolated from nosocomial infections, with resistance rates of $64 \%$ to meropenem, $63.8 \%$ to piperacillin/tazobactam, $63.4 \%$ to amikacin and $55.8 \%$ to ceftazidime [5]. The USA's national nosocomial infections surveillance system (NNIS) showed that in 2003 P. aeruginosa accounted for $18.1 \%$ of the cases of pneumonia, 9.5\% of surgical wound infections and $16.3 \%$ of urinary infections among ICU patients and increases of $17.7 \%, 27.3 \%$ and $26.4 \%$ in resistance to imipenem, quinolone and third-generation cephalosporin, respectively, in that country [6].

The factors that predict a fatal outcome vary among studies and include septic shock, pneumonia, advanced age, severity of the underlying disease, use of inappropriate antimicrobials and emergence of antimicrobial resistance during treatment [7-11]. Rello et al. [12] estimated a mortality rate of $13.5 \%$ attributable to pneumonia associated with mechanical ventilation caused by P. aeruginosa. A systematic review of the literature emphasized that the previous use of antibiotics is the principal risk factor for acquiring multiresistant $P$. aeruginosa [13].

Received on 29 April 2008; revised 19 November 2008.

Address for correspondence: Dr. Millena R. Pinheiro. Rua José Carvalheira, 392/1003- Tamarineira, Recife, PE, Brazil. Zip Code: 52051-060. Phone/fax: +55 81 32655811/ +55 81 21268527. E-mail: millena_pinheiro@hotmail.com.

The Brazilian Journal of Infectious Diseases

2008;12(6):509-515. (C) 2008 by The Brazilian Journal of Infectious Diseases and Contexto Publishing. All rights reserved.
Timely recognition of variables presenting a risk of death among patients infected with $P$. aeruginosa may lead to changes in patient management by the medical team, with earlier and better-targeted interventions. The aim of this study was to define risk factors associated with unfavorable clinical outcomes in $P$. aeruginosa infections among critically ill patients, with special emphasis on bacterial resistance and antimicrobial treatment.

\section{Material and Methods}

This retrospective case-control study comprised 131 patients admitted to an ICU from four tertiary-level hospitals in Recife, Brazil, between January 2006 and August 2007. Cases presented positive cultures for $P$. aeruginosa and criteria of infection based on NNIS criteria of the Centers for Disease Control [14] and progressed to death within 30 days. Controls presented positive cultures for $P$. aeruginosa and the same NNIS criteria for infection and were discharged within 30 days of the diagnosis. Only the first episode of infection per site and per patient was considered. Patients under 16 years and those with cystic fibrosis were excluded. This study was approved by the Ethics Committee of the Oswaldo Cruz University Hospital.

P. aeruginosa was identified using conventional methods. The susceptibility to antibiotics was determined using the Kirby-Bauer disc diffusion method, in accordance with NCCLS, 2004 [15]. Screening for resistance to polymyxin B used the protocol described by Gales [16].

\section{Definition}

P. aeruginosa was defined as resistant (resistance in vitro to at least one of the anti-Pseudomonas antibiotics: piperacillin, ciprofloxacin, ceftazidime or imipenem), multiresistant (resistance to at least three classes of anti-Pseudomonas antibiotics) or panresistant (resistance to carbapenems and to all the anti-Pseudomonas antibiotics available, with the exception of polymyxin B). 
Empirical antibiotic therapy was the therapy given between 8h before the index blood culture was drawn and the time antibiotic susceptibility testing results became available. Empirical antibiotic therapy was considered appropriate if it included antipseudomonal antibiotics to which the isolate displayed in vitro susceptibility. Neither aminoglycoside nor aztreonam monotherapy were considered adequate therapy for $P$. aeruginosa bacteremia [17].

Potential risk factors for death among critically ill patients with $P$. aeruginosa infection were identified by means of multivariate analysis. The chi-squared or Fisher test was used for categorical variables. Continuous variables were tested using Student's t or the Mann-Whitney test. Variables for which there was a p value $<0.20$ in bivariate analysis were included in the logistic regression model for multivariate analysis. Epi Info version 6.04 and SPSS version 8.0 were used for analysis.

\section{Results}

A total of 131 episodes of $P$. aeruginosa infection were analyzed. The mortality rate was 51.1\% (67 patients). Patient characteristics are shown in Table 1.

Antibiotic susceptibility was generally less than $70 \%$ for piperacillin/tazobactam, imipenem, meropenem and amikacin and $31(23.7 \%)$ were resistant to all the agents used in the tests, except polymyxin (Table 2).

The antimicrobial agents most frequently used and their combinations are shown in Table 3. Use of aminoglycosides predominated in both empirical and definitive combination therapy.

Seventy-one patients received monotherapy (56.3\%), fiftyfive $(43.7 \%)$ were treated with two anti-Pseudomonas drugs and five patients did not receive any antibiotic treatment. The antimicrobial resistance pattern of $P$. aeruginosa was unrelated to mortality, but was inversely related to the adequacy of the initial antimicrobial therapy (Table 4).

There was greater mortality among the patients who received combination antibiotic therapy, although there were no statistical differences between the APACHE II scores for the groups with monotherapy or combination therapy. Empirical treatment presented a greater adequacy when two or more drugs were used (33 out of 54 versus 43 out of $71 ; \mathrm{p}=$ 0.70). The infection site for which combination therapy was most used was the bloodstream.

Forty-seven patients (37.3\%) received inappropriate empirical treatment, although this did not result in greater mortality $(p=0.96)$. Seventy-nine patients $(62.7 \%)$ received appropriate antibiotics within 24 hours of collection of cultures, while fifteen (11.9\%) received appropriate antibiotics only after receiving their results. Thirty-two patients did not receive appropriate definitive treatment (25.4\%). The mean duration of the antibiotic treatment was $13.8 \pm 7.8$ days. Bivariate analysis identified age over 60 years, APACHE II on admission and septic shock as factors associated with death (Table 5). With regard to laboratory parameters, both serum albumin $(\mathrm{OR}=0.26 ; \mathrm{p}=0.001)$ and blood $\mathrm{pH}(\mathrm{p}=0.061 ; \mathrm{R}=$ 0.06 ) showed associations with mortality, whereas neither the leukocytes $(p=0.724)$ nor creatinine $(p=0.555)$ showed any relationship with mortality.

There was a greater frequency of septic shock in the group with inappropriate therapy, both for empirical therapy (29 out of 47 versus 36 out of 79; $\mathrm{p}=0.11$ ) and for definitive therapy (21 out of 32 versus 44 out of $94 ; p=0.10$ ).

Multivariate regression analysis identified age (OR 1.04), serum albumin (OR 0.32) and septic shock (OR 15.4) as factors independently associated with death.

\section{Discussion}

In the light of uncertainties about treatment of Pseudomonas infections within the present-day scenario of multiresistance, the authors of the study set out to determine what factors relating to antimicrobial therapy might have an impact on mortality among patients with $P$. aeruginosa infection. For this purpose, they conducted a case-control study in which all possible factors relating to death among patients infected with this pathogen were analyzed. The characteristics of the antimicrobial therapy were included as risk factors. The factors clearly related to death were the more classical ones, such as age, septic shock and serum albumin $[2,17,18,19,20]$. However, several therapy-related findings were curious, producing some surprising revelations. The first was the high frequency of inappropriate initial treatment, which totaled 47 cases (37.3\%). The second was that the adequacy of the treatment did not make any difference to eventual mortality. The third was that combination therapy (regardless of whether it was empirical or definitive) may have been associated with greater mortality (OR 1.97, $\mathrm{p}=0.09$; OR 2.0, $\mathrm{p}$ $=0.08$ ), despite not attaining statistical significance.

The high frequency of inappropriate initial treatment was directly related to bacterial resistance. In other words, within the scenario of multiresistance, it is more difficult to institute the appropriate treatment. This may be explained by a lack of knowledge among the medical team regarding bacterial resistance patterns within the institution, or it may be due to fear of the indiscriminate empirical use of broad-spectrum antibiotics or even of those with a more specific spectrum (such as polymyxin). However, as with the results presented by Osih [17], inadequacy did not produce any difference in mortality. This may be due to the difference between the in vitro and in vivo effects of the antimicrobial agents in relation to $P$. aeruginosa, such that the treatment may be successful even in the presence of infections caused by multiresistant or panresistant strains. Another argument would be that patients with $P$. aeruginosa infections that appear at an advanced stage of hospitalization have underlying diseases that would lead to death irrespective of the infection, and thus $P$. aeruginosa would not be the cause of death. Lastly, there could be other infectious agents present (the observed frequency of polymicrobial infection is $28.2 \%$ ) which may have been treated through the therapy used. 
Table 1. Characteristics of the 131 patients with Pseudomonas aeruginosa infection.

\begin{tabular}{|c|c|c|}
\hline & $\mathbf{N}$ & $\%$ \\
\hline Age (mean, yr) & $64.2+18.4$ & \\
\hline APACHE II (on admission) & $18.0+7.6$ & \\
\hline SOFA(on admission) & $6.5+3.3$ & \\
\hline Duration of hospitalization before infection (days) & - & - \\
\hline Sex (male) & 66 & 50.4 \\
\hline \multicolumn{3}{|l|}{ Origin } \\
\hline Community & 58 & 44.3 \\
\hline Nosocomial & 70 & 53.4 \\
\hline Home care & 3 & 2.3 \\
\hline \multicolumn{3}{|l|}{ Comorbidities } \\
\hline Systemic arterial hypertension & 73 & 56.6 \\
\hline Heart disease/ coronary disease & 40 & 31.0 \\
\hline Diabetes mellitus & 38 & 29.5 \\
\hline Cerebrovascular disease & 37 & 28.7 \\
\hline Surgery & 25 & 19.4 \\
\hline Chronic kidney failure & 23 & 18.0 \\
\hline Neoplasia & 16 & 12.4 \\
\hline Immunodepression & 15 & 11.6 \\
\hline COPD & 12 & 9.3 \\
\hline \multicolumn{3}{|l|}{ Infection site } \\
\hline Lung & 86 & 65.6 \\
\hline Bloodstream & 24 & 18.3 \\
\hline Urinary tract & 15 & 11.5 \\
\hline Surgical site & 5 & 3.8 \\
\hline Skin/ soft tissue & 1 & 0.8 \\
\hline Central nervous system involvement & 96 & 73.3 \\
\hline Use of mechanical ventilation & 121 & 92.4 \\
\hline Use of central catheter & 130 & 99.2 \\
\hline Use of bladder catheter & 122 & 93.1 \\
\hline Use of parenteral nutrition & 12 & 9.2 \\
\hline Polymicrobial infection & 37 & 28.2 \\
\hline Hemodialysis & 38 & 29.0 \\
\hline Septic shock & 66 & 50.4 \\
\hline Activated drotrecogin-alpha & 1 & 0.9 \\
\hline
\end{tabular}

Table 2. Pattern of susceptibility to antimicrobial agents among Pseudomonas aeruginosa strains.

\begin{tabular}{lcc}
\hline Sensitivity pattern & Strains tested (N) & Sensitive (\%) \\
\hline Polymyxin B & 24 & 100 \\
Piperacillin-tazobactam & 101 & 64.3 \\
Imipenem & 126 & 62.6 \\
Amikacin & 124 & 61.2 \\
Meropenem & 116 & 60.3 \\
Ciprofloxacin & 127 & 48.0 \\
Aztreonam & 128 & 46.8 \\
Gentamicin & 128 & 44.5 \\
Ceftazidime & 76 & 43.4 \\
Cefepime & 116 & 39.6 \\
\hline
\end{tabular}


Table 3. Antimicrobial agents and combinations most used for empirical and definitive therapy.

\begin{tabular}{lc}
\hline Antibiotic treatment & No. of treatments \\
\hline Empirical therapy & \\
Monotherapy & \\
Meropenem/Imipenem & $32(33.3 \%)$ \\
Piperacillin/tazobactam & $18(18.7 \%)$ \\
Cefepime & $4(4.2 \%)$ \\
Polymyxin B & $3(3.1 \%)$ \\
Combination therapy & \\
Piperacillin/tazobactam + aztreonam & $9(9.4 \%)$ \\
Piperacillin/tazobactam + amikacin & $5(5.2 \%)$ \\
Meropenem/Imipenem + amikacin & $8(8.4 \%)$ \\
Cefepime + amikacin & $4(4.2 \%)$ \\
Cefepime + ciprofloxacin & $4(4.2 \%)$ \\
Polymyxin B + carbapenem & $3(3.1 \%)$ \\
Imipenem + ciprofloxacin & $3(3.1 \%)$ \\
Piperacillin/tazobactam + ciprofloxacin & $3(3.1 \%)$ \\
Definitive therapy & \\
Monotherapy & \\
Meropenem/Imipenem & $35(39.2 \%)$ \\
Piperacillin/tazobactam & $16(18.0 \%)$ \\
Polymyxin B & $7(7.8 \%)$ \\
Cefepime & $6(6.8 \%)$ \\
Combination therapy & \\
Piperacillin/tazobactam + amikacin & $6(6.8 \%)$ \\
Piperacillin/tazobactam + aztreonam & $6(6.8 \%)$ \\
Imipenem + amikacin & $5(5.6 \%)$ \\
Polymyxin B + carbapenem & $4(4.5 \%)$ \\
Imipenem + ciprofloxacin & $4(4.5 \%)$ \\
\hline
\end{tabular}

Table 4. Association between antimicrobial resistance pattern and adequacy of initial empirical therapy.

\begin{tabular}{lccccc}
\hline Resistance pattern & \multicolumn{4}{c}{ Initial empirical therapy } \\
\cline { 2 - 3 } & \multicolumn{2}{c}{ Adequate } & & & \multicolumn{2}{c}{ Inadequate } \\
\cline { 2 - 3 } \cline { 5 - 6 } & $\mathbf{N}$ & $\mathbf{N}$ & & $\%$ \\
\hline $\begin{array}{l}\text { Resistant } \\
\text { (61 strains) }\end{array}$ & 51 & 87.9 & 7 & 12.1 \\
$\begin{array}{l}\text { Multiresistant } \\
\text { (39 strains) }\end{array}$ & 24 & 64.9 & 13 & 35.1 \\
$\begin{array}{l}\text { Panresistant } \\
\text { (31 strains) }\end{array}$ & 4 & 12.9 & 27 & 87.1 \\
\hline
\end{tabular}

The possible role of the combination therapy as a risk factor for death was the most surprising finding from the present study. This is at odds with the findings of several authors who have evaluated treatments for $P$. aeruginosa infection $[19,21,22]$.

One might ask whether the combination therapy had been indicated for the more severe cases or those with a greater chance of immunosuppression, who would therefore be at greater risk of death. However, on comparing the APACHE II scores for the two groups, we did not find any differences. Nor did we find any differences in outcome from infections in the presence of immunosuppression. On the other hand, combination therapy was used more often in cases of infection of the bloodstream, generally relating to bacteremia, and thus with a greater risk of death $[1,8,23,24]$. Moreover, since aminoglycosides were the antimicrobial agents most used in associations and, over the period of this study there was no consensus regarding their use for limited periods, the cause of death could be attributable to their adverse effects. Most of our patients were elderly and kidney failure may have worsened the prognosis for the individuals treated with this medication $[9,25]$. Obritsch et al. studied the emergence of 
Table 5. Prognostic factors for hospital mortality: bivariate analysis.

\begin{tabular}{|c|c|c|c|c|c|c|c|}
\hline \multirow[t]{3}{*}{ Factors } & \multicolumn{4}{|c|}{ Outcome } & \multirow{3}{*}{\multicolumn{2}{|c|}{ OR(CI) }} & \multirow[t]{3}{*}{ p value } \\
\hline & \multicolumn{2}{|c|}{ Death } & \multicolumn{2}{|c|}{ Discharge/cure } & & & \\
\hline & $\mathbf{N}$ & $\%$ & $\mathbf{N}$ & $\%$ & & & \\
\hline \multicolumn{8}{|l|}{ Age (years) } \\
\hline Less than 60 years & 19 & 39.6 & 29 & 60.4 & 1.0 & - & \\
\hline More than 60 years & 48 & 57.8 & 35 & 42.2 & 2.09 & $(0.95-4.65)$ & 0.06 \\
\hline \multicolumn{8}{|l|}{ Gender } \\
\hline Female & 34 & 52.3 & 31 & 47.7 & 1.0 & - & \\
\hline Male & 33 & 50.0 & 33 & 50.0 & 0.91 & $(0.43-1.93)$ & 0.928 \\
\hline \multicolumn{8}{|c|}{ Fever present at time of diagnosis } \\
\hline No & 29 & 51.8 & 27 & 48.2 & 1.0 & - & \\
\hline Yes & 38 & 50.7 & 37 & 49.3 & 0.96 & $(0.45-2.04)$ & 0.96 \\
\hline \multicolumn{8}{|c|}{ Central nervous system involvement } \\
\hline No & 13 & 37.1 & 22 & 62.9 & 1.0 & - & \\
\hline Yes & 54 & 56.3 & 42 & 43.7 & 2.18 & $(0.91-5.25)$ & 0.082 \\
\hline \multicolumn{8}{|l|}{ Immunosuppression } \\
\hline No & 49 & 50.5 & 48 & 49.5 & 1.0 & - & \\
\hline Yes & 18 & 52.9 & 16 & 47.1 & 1.10 & $(0.47-2.61)$ & 0.964 \\
\hline \multicolumn{8}{|c|}{ Association with pneumonia } \\
\hline No & 19 & 47.5 & 21 & 52.5 & 1.0 & - & \\
\hline Yes & 47 & 58.0 & 34 & 42.0 & 1.53 & $(0.66-3.54)$ & 0.368 \\
\hline \multicolumn{8}{|c|}{ Association with septic shock } \\
\hline No & 16 & 24.6 & 49 & 75.4 & 1.0 & - & \\
\hline Yes & 51 & 77.3 & 15 & 22.7 & 10.4 & $(4.3-25.7)$ & 0.000 \\
\hline \multicolumn{8}{|l|}{ Resistance pattern } \\
\hline Resistant & 32 & 52.5 & 29 & 47.5 & 1.0 & - & \\
\hline Multiresistant & 16 & 41.0 & 23 & 59.0 & 0.63 & $(0.27-1.42)$ & 0.266 \\
\hline Panresistant & 19 & 61.3 & 12 & 38.7 & 1.43 & $(0.59-3.46)$ & 0.421 \\
\hline \multicolumn{8}{|c|}{ Previous use of antibiotic } \\
\hline No & 08 & 42.1 & 11 & 57.9 & 1.0 & - & \\
\hline Yes & 59 & 52.7 & 53 & 47.3 & 1.53 & $(0.52-4.60)$ & 0.545 \\
\hline \multicolumn{8}{|c|}{ Delayed empirical therapy } \\
\hline No & 55 & 56.7 & 42 & 43.3 & 1.0 & - & \\
\hline Yes & 11 & 37.9 & 18 & 62.1 & 0.47 & $(0.18-1.19)$ & 0.117 \\
\hline \multicolumn{8}{|c|}{ Combination empirical therapy } \\
\hline No & 32 & 45.1 & 39 & 54.9 & 1.0 & - & \\
\hline Yes & 34 & 61.8 & 21 & 38.1 & 1.97 & $(0.90-4.35)$ & 0.091 \\
\hline \multicolumn{8}{|c|}{ Adequate empirical therapy } \\
\hline No & 24 & 51.1 & 23 & 48.9 & 1.0 & - & \\
\hline Yes & 42 & 53.2 & 37 & 46.1 & 1.09 & $(0.49-2.41)$ & 0.964 \\
\hline \multicolumn{8}{|c|}{ Combination definitive therapy } \\
\hline No & 33 & 45.2 & 40 & 54.8 & 1.0 & - & \\
\hline Yes & 33 & 62.3 & 20 & 37.7 & 2.00 & $(0.91-4.43)$ & 0.086 \\
\hline \multicolumn{8}{|c|}{ Adequate definitive therapy } \\
\hline No & 17 & 53.1 & 15 & 46.9 & 1.0 & - & \\
\hline Yes & 49 & 52.1 & 45 & 47.9 & 0.96 & $(0.40-2.33)$ & 0.914 \\
\hline \multicolumn{8}{|l|}{ Catheter removal } \\
\hline No & 14 & 38.9 & 22 & 61.1 & 1.0 & - & \\
\hline Yes & 12 & 60.0 & 08 & 40.0 & 2.35 & $(0.77-7.20)$ & 0.133 \\
\hline
\end{tabular}


resistance to Pseudomonas during a ten year-period and observed greater rates of resistance in the combination therapy containing ciprofloxacin, especially when associated with cefepime or imipenem. The best combination was aminoglycoside or fluorquinolone in association with piperacillin/tazobactam [26]. A survey conducted in two local hospitals by Figueiredo et al. [27] found that the rate of resistance to ciprofloxacin was high, such that it presented activity against only $49.7 \%$ of all of the strains of $P$. aeruginosa and the susceptibility to aminoglycosides was $59.4 \%$ for amikacin and 48.6\% for gentamicin. Magalhães et al. carried out a molecular analysis on 48 strains of $P$. aeruginosa from Recife/Brazil. They demonstrated that $62.5 \%$ of the strains produced metallo- $\beta$-lactamase (SPM-1) with a broad resistance profile, such that they were only susceptible to polymyxin and aztreonam [28]. The best association of antimicrobial agents for treating infections due to $P$. aeruginosa is still a matter of controversy.

The indication that delayed therapy could protect against death, albeit without clinical significance (OR 0.47; p = 0.112), was surprising. This may have resulted from delayed therapy for infections of lesser severity. Identifying the time at which the appropriate therapy becomes critical to the outcome for the patient is particularly important because of the delicate balance that exists between the beneficial effect of the appropriate therapy on the patient's survival and development of antimicrobial resistance through excessive use of broadspectrum antibiotics [17].

The present study is subject to various criticisms, such as the small number of cases and the use of retrospective data from medical records. Nevertheless, methodological precautions were taken to ensure the quality of the study. These included the use of very clear definitions for the therapy (empirical, definitive, inappropriate, delayed and combination), definitions for infections according to the NNIS criteria and use of logistic regression [22,29]. Several findings suggest that its results are coherent, such as the inversely proportional relationship between therapeutic adequacy and the likely degree of resistance; the sensitivity pattern for P. aeruginosa, which was very similar to that reported in the MYSTIC study [5] and another study conducted recently in the state of Pernambuco [27]; and, finally, the finding of greater mortality among shock patients and elderly individuals, which is compatible with the literature on severe infections.

In conclusion, the risk factors for death in $P$. aeruginosa infections are similar to those of other severe infections, i.e. age, presence of septic shock and low serum albumin levels [2,3,18]. There was a high frequency of inappropriate treatment, but this does not seem to have influenced the outcome. Thus, treatment should always be attempted. There were indications that combination therapy may be associated with greater mortality.

\section{Acknowledgements}

We would like to thank Dr Luiz Loureiro Santana and Dr Antonio Jaime da Fonte for permission to access the medical files.

\section{References}

1. Baltch A.L., Smith R.P. Combinations of antibiotics against Pseudomonas aeruginosa. Am J Med 1985;79:8-16.

2. Aliaga L., Mediavilla J.D., Cobo F. A clinical index predicting mortality with Pseudomonas aeruginosa bacteremia. J Med Microbiol 2002;51:615-9.

3. Kang C., Kim S.H. Bloodstream infections caused by antibioticresistant Gram-negative bacilli: risk factors for mortality and impact of inappropriate initial antimicrobial therapy on outcome. Antimicrob Agents and Chemoth 2005;49:760-6.

4. Sader H.S., Jones R.N., Gales A.C. SENTRY Antimicrobial Surveillance Program report: Latin American and Brazilian results for 1997 through 2001. Braz J Infect Dis 2004;8:25-79.

5. Kiffer C., Hsiung A., Oplustil C. Antimicrobial susceptibility of Gram-negative bacteria in Brazilian hospitals: the MYSTIC Program Brazil 2003. Braz J Infect Dis 2005;9:216-24.

6. Gaynes R., Edwards J.R. National Nosocomial Infections Surveillance System. Overview of nosocomial infections caused by Gram-negative bacilli. Clin Infect Dis 2005;41:848-54.

7. Bodey G.P., Jadeja L., Elting L. Pseudomonas bacteremia. Retrospective analysis of 410 episodes. Arch Intern Med 1985;145:1621-9.

8. Vidal F., Mensa J., Almela M., et al. Epidemiology and outcome of Pseudomonas aeruginosa bacteremia, with special emphasis on the influence of antibiotic treatment. Analysis of 189 episodes. Arch Inter Med 1996;156:2121-6.

9. Chen S.C., Lawrence R.H., Byth K. Pseudomonas aeruginosa bacteremia. Is pancreatobiliary disease a risk factor? Med J Aust 1993;159:592-7.

10. Kuikka A., Valtonen V.V. Factors associated with improved outcome of Pseudomonas aeruginosa bacteremia in a Finnish University Hospital. Eur J Clin Microbiol Infect Dis 1998;17:701-8.

11. Zelenitsky S.A., Harding G.K.M., Sun S. Treatment and outcome of Pseudomonas aeruginosa bacteremia: an antibiotic pharmacodynamic analysis. J Antimicrob Chemother 2003;52:668-74.

12. Rello J., Rue M., Jubert P., et al. Survival in patients with nosocomial pneumonia: impact of the severity of illness and the etiologic agent. Crit Care Med 1997;25:1862-7.

13. Falagas M.E., Kopterides P. Risk factors for the isolation of multidrug-resistant Acinetobacter baumannii and Pseudomonas aeruginosa: a systematic review of the literature. J Hosp Infect 2006;64:7-15.

14. Horan T.C., Gaynes R.P. Surveillance of nosocomial infections. In: Hospital Epidemiology and Infection Control, $3^{\text {rd }}$ ed., Mayhall CG, editor. Philadelphia: Lippincott Williams \& Wilkins, 2004:1659-1702.

15. National Committee for Clinical Laboratory Standards. Performance standards for antimicrobial disk susceptibility test. Fourteenth Informational Supplement. Wayne, PA: NCCLS; 2004.

16. Gales A.C., Reis A.O., Jones R.N. Contemporary assessment of antimicrobial susceptibility testing methods for Polymyxin B and Colistin: review of available interpretative criteria and quality control guidelines. J Clin Microbiol 2001;39:183-90.

17. Osih R.B., McGregor J.C., Rich S.E. Impact of empiric antibiotic therapy on outcomes in patients with Pseudomonas aeruginosa bacteremia. Antimicrob Agents Chemother 2006:901-6.

18. Leary M.J., Koll M., Ferguson C.N. Liver albumin synthesis in sepsis in the rat: influence of parenteral nutrition, glutamine and growth hormone. Clin Sci 2003;105:691-8.

19. Micek S.T., Lloyd A.E., Ritchie D.J. Pseudomonas aeruginosa bloodstream infection: importance of appropriate initial antimicrobial treatment. Antimicrob Agents Chemother 2005;49:1306-11.

20. Zavascki A.P., Barth A.L., Gonçalves A.L.S. The influence of metallo-â-lactamase production on mortality in nosocomial Pseudomonas aeruginosa infections. J Antimicrob Chemot 2006; 58 :387-92. 
21. Chamot E., El Amari E.B., Rohner P. Effectiveness of combination antimicrobial therapy for Pseudomonas aeruginosa bacteremia. Antimicrob Agents Chemother 2003;47:2756-64.

22. Gamacho-Montero J., Sa-Borges M., Sole-Violan J. Optimal management therapy for Pseudomonas aeruginosa ventilatorassociated pneumonia: an observational, multicenter study comparing monotherapy with combination antibiotic therapy. Crit Care Med 2007;35:1888-95.

23. Norrby S.R., Finch R.G., Glauser M. Monotherapy in serious hospital-acquired infections: a clinical trial of ceftazidime versus imipenem/cilastatin. European Study Group. J Antimicrob Chemother 1993;31:927-37.

24. Siegman-Igra Y., Ravona R., Primerman H., Giladi M. Pseudomonas aeruginosa bacteremia: an analysis of 123 episodes, with particular emphasis on the effect of antibiotic therapy. Int J Infec Dis 1998;2:211-5.
25. Rolston K.V., Bodey G.P. Pseudomonas aeruginosa infection in cancer patients. Cancer Invest 1992;10:43-59.

26. Obritsch M.D., Fish D.N., MacLaren R. National surveillance of antimicrobial resistance in Pseudomonas aeruginosa isolates obtained from intensive care unit patients from 1993 to 2002. Antimicrob Agents Chemother 2004:4606-4610.

27. Figueiredo E.A.P., Ramos H., Maciel M.A.V., et al. Pseudomonas aeruginosa: freqüência de resistência a múltiplos fármacos e resistência cruzada entre antimicrobianos no Recife/PE. Rev Bras Terap Intensiva 2007; 19:421-7.

28. Magalhaes V., Lins A.K., Magalhaes M. Metallo-beta-lactamase producing Pseudomonas aeruginosa strains isolated in hospitals in Recife, PE, Brazil. Braz J Microbiol 2005;36:123-5.

29. National Nosocomial Infections Surveillance. Report, data summary from January 1992 through June 2004, issued October 2004. Am J Infect Control 2004;32:470-85. 